\title{
Reevaluación del potencial geotérmico de los granitos de Galicia en base a cartografía geoquímica y radiológica.
}

\section{Re-evaluation of the geothermal potential of the granites of Galicia based on geochemical and radiological cartography}

\section{SANJURJO-SÁNCHEZ, J. ${ }^{1}$, BARRIENTOS RODRÍGUEZ, V. ${ }^{1}$}

${ }^{1}$ Instituto Universitario de Xeoloxía "Isidro Parga Pondal". Universidade da Coruña, Campus de Elviña, 15071 A Coruña, España.

\section{Resumen}

El flujo de calor en la corteza superior determina la posibilidad de utilizar la energía geotérmica en una determinada zona. Este flujo es muy heterogéneo por lo que el aprovechamiento de la energía geotérmica es muy variable y requiere un estudio de cada zona. Donde no existen condicionantes geológicos que produzcan un flujo de calor anómalo y elevado, el aprovechamiento de esta energía es más limitado, dependiendo de las propiedades de las rocas del substrato, pero aun así enormemente variable. Los estudios realizados en Galicia hasta la fecha indican que el aprovechamiento geotérmico en esta comunidad es limitado debido a la ausencia de anomalías y a la litología, existiendo un potencial geotérmico bajo. 
Sin embargo, los principales estudios orientados a este fin, aun considerando información geológica, han sido genéricos, imprecisos y conservadores. La existencia de nuevos datos geoquímicos y de radiación gamma permite una reevaluación de estos estudios. Esta reevaluación muestra un potencial mayor del estimado inicialmente, lo que sugiere la necesidad de realizar estudios más detallados de la geoquímica de las rocas para obtener una evaluación más precisa y exacta, algo necesario para un desarrollo adecuado del aprovechamiento de esta energía en la comunidad.

PALABRAS CLAVE: Geotermia, Bomba de calor, Energía, Radiación gamma, Radioisótopos.

\begin{abstract}
The heat flux in the upper crust determines the availabili of geothermal energy resources whose use depending on the properties of the rocks substrate. In Galicia the geothermal exploitation is limited, because the low potential. However the existence of new geochemical and gamma radiation allows a re-evaluation carrying out more detailed studies of rock geochemistry to obtain a more precise evaluatón for an adequate development of the use of this energy.
\end{abstract}

KEY WORDS: Geothermal energy, heat pump, Energy, Gamma radiation, Radioisotopes. 


\section{INTRODUCCIÓN}

Se denomina energía geotérmica a la energía calorífica almacenada en la corteza superior de la Tierra y geotermia a la disciplina que estudia esta energía, su origen distribución y posible uso. El aprovechamiento energético de este calor implica recuperarlo de aguas subterráneas o de las rocas del subsuelo y utilizarlo para generar electricidad o usar el propio calor con otros fines. La distribución del calor en la corteza superior es heterogénea debido a la Tectónica de Placas y a las características de los distintos tipos de roca. En términos globales, se puede decir que la mayor parte del volumen de la Tierra se encuentra a temperaturas superiores a $1000^{\circ} \mathrm{C}$, mientras que una parte ínfima, la superficial que supone en torno al $0,1 \%$, se encuentra a menos de $100^{\circ} \mathrm{C}$. Ese calor fluye a la superficie por convención, conducción y radiación térmica de un modo desigual. La principal fuente de este calor es la desintegración de radioisótopos primordiales, principalmente del potasio-40 $\left({ }^{40} \mathrm{~K}\right)$ y de las series del uranio $\left({ }^{235} \mathrm{U},{ }^{238} \mathrm{U}\right)$ torio $\left({ }^{232} \mathrm{Th}\right)$, aunque existen otras fuentes como el calor residual liberado durante la formación de la Tierra, o el generado, entre otros, por los movimientos entre capas (Pollack et al. 1993; Pous y Jutglar, 2004; Orche García, 2011).

La mayor parte de la superficie terrestre tiene un flujo de calor muy bajo (50-100 $\mathrm{mW} / \mathrm{m}^{2}$ ) siendo el valor medio del gradiente geotérmico en la corteza de $2-4^{\circ} \mathrm{C}$ cada 100 $\mathrm{m}$ de profundidad. Sin embargo, existen zonas del planeta en la que ese flujo es mayor, registrándose un gradiente de hasta 15-50 ${ }^{\circ} \mathrm{C}$ cada $100 \mathrm{~m}$, debido a causas geológicas. Estas son zonas de bordes divergentes entre placas tectónicas, zonas de bordes conver- gentes, zonas de actividad distensiva en una placa (con adelgazamiento de la corteza) y puntos calientes intraplaca. Por ello, el uso de la energía geotérmica está limitado por las condiciones geológicas. Además, el progreso técnico en la eficiencia y el uso del calor hace que evaluar el potencial geotérmico sea un proceso complejo y cambiante. Aun así, el uso de la energía geotérmica ha ido creciendo constantemente, y aunque su uso comenzó hace ya 100 años, su crecimiento en los últimos 20 años ha sido enorme. Este incremento se refleja en su uso en producción de electricidad, que se ha visto incrementado en un 16\% desde el año 1999 al 2004 (un $3 \%$ anual) y un $43 \%$ en el uso directo de calor (un 7,5\% al año). El incremento anual en su uso ha sido del 5,5\% entre los años 1980 a 2010 (ACLUXEGA, 2012, GEOPLAT, 2015). Sin embargo, este desarrollo varía enormemente en función del país debido a condicionantes geológicos, tecnológicos y financieros. Mientras que en muchos países de Europa ha habido un gran desarrollo, en España éste ha sido discreto (ACLUXEGA, 2012, GEOPLAT, 2015). A pesar de esto el European Geothermal Energy Council (EGEC) ha establecido una serie de objetivos hasta el año 2050 que se resumen en los siguientes (Orche García, 2011):

- Objetivo 2020: establecer base de industria geotérmica europea;

- Objetivo 2030: Hacia una fuente de electricidad competitiva;

- Objetivo 2050: Una parte sustancial de suministro base de electricidad.

En España, existiendo excepciones, la mayor parte del territorio tiene un flujo de calor muy bajo. Aunque el concepto de recurso geotérmico es muy amplio, se pueden clasificar estos recursos en función de su nivel térmico en base a unos límites estableci- 
dos por la Plataforma Tecnológica Española de Geotermia (GEOPLAT) en recursos de alta entalpía $\left(\mathrm{T}>150^{\circ} \mathrm{C}\right)$, de media entalpía $\left(\mathrm{T}=100-150^{\circ} \mathrm{C}\right)$, baja entalpía $\left(\mathrm{T}=30-100^{\circ}\right)$ y muy baja entalpía $\left(\mathrm{T}<30^{\circ} \mathrm{C}\right)$. Las posibilidades energéticas y técnicas de aprovechamiento varían en función del tipo de recurso o del yacimiento geotérmico, entendiéndose como tal el volumen de roca permeable y caliente de la cual se puede extraer el calor utilizando como vector de transporte el fluido que contiene o uno introducido artificialmente. Este fluido suele ser agua.

La evaluación de los recursos se puede realizar de varias formas, pero las evaluaciones realizadas a nivel mundial dejan patente que un mayor conocimiento geológico proporciona una mayor confianza en las estimaciones. La heterogeneidad de los materiales geológicos y la cantidad de factores que condicionan muchos aspectos del flujo de calor limitan la posibilidad de obtener una buena estimación del potencial geotérmico, que requiere un estudio lo más detallado y local posible para obtener una mayor exactitud y precisión. En este sentido, se han realizado varias estimaciones de los recursos geotérmicos a nivel español. La amplitud del territorio abarcado dificulta una estimación exacta, de modo que para obtenerla se han utilizado valores promedio, tabulados, por convenio, o se han realizado aproximaciones teóricas. Esto hace que salvo para el caso de algunas comunidades autónomas (como Cataluña y Extremadura) las estimaciones sean cuanto menos imprecisas.

Las principales evaluaciones realizadas para el territorio español son dos, aunque hay algunos hitos históricos que permiten explicar la evolución de la geotermia en España y que se resumen en el siguiente cronograma histórico (Orche García, 2011, IDEA, 2011):
- Antes de 1974: estudios puntuales del IGME (Lanzarote, 1948-1949).

- 1974-1975: Inventario Nacional de Manifestaciones Geotérmicas (IGME).

- 1980-1984: Estudios de factibilidad térmico-económica (IGME y E.N. ADA$\mathrm{RO}$ ),

- 1987-1990: Estudio de la Comunidad de Madrid

- 2008: I Congreso de Energía Geotérmica en la Edificación y la Industria, Madrid. (GEOENER): V GEOENER-2017.

- 2009: Establecimiento de la Plataforma Tecnológica Española de Geotermia (GEOPLAT).

- 2012: Presentación de un Atlas Geotérmico de Galicia (III GEOENER 2012) que no es de acceso público.

- Plan de Energías Renovables de España 2005-2010 (PER 2005-2010) que no incluye recursos geotérmicos.

- Plan de Acción Nacional de Energías Renovables en España (PANER) para 2011-2020: APPA y GEOPLAT incluyendo el desarrollo de Sistemas Geotérmicos Estimulados (EGS)

\subsection{Evaluación del potencial Geotérmico de Galicia}

Las dos evaluaciones del potencial geotérmico realizadas para todo el territorio nacional arrojan resultados relativamente diferentes. En 1975, el IGME realizó el primer estudio sobre geotermia en España (IGME, 1975), publicando como resultado un mapa de indicios geotérmicos y otro de áreas geotérmicas en donde se establece un escaso interés en la mayor parte del territorio de Galicia y solo un posible interés con almacenes de media temperatura (me- 
dia-baja entalpía) en zonas del Oeste de la provincia de Ourense y del centro de la de Pontevedra.

A parte de este estudio, se realiza un estudio más detallado de Galicia que dio lugar al informe titulado "Estudio de las manifestaciones termales de Galicia, orientadas a su posible explotación como recursos geotérmicos" publicado en el año 1980 (IGME, 1980). En este informe se estudian la temperatura, composición y condiciones de surgencia superficial de la mayor parte de las aguas termales de Galicia. En las conclusiones del informe, se destaca cierta problemática para su explotación como la falta de permeabilidad de los almacenes geotérmicos. El estudio recomienda continuar la investigación con estudios más detallados y específicos, particularmente en el sector occidental de la provincia de Orense y parte $\mathrm{N}$ de la de Pontevedra, donde se encuentran la mayor parte de las manifestaciones termales, que además tienen las mayores temperaturas de surgencia y del almacén geotérmico. Así mismo, se justifica su estudio por ser la región más densamente poblada, aspecto que se ha incluso incrementado.

La segunda evaluación realizada a nivel de España, correspondiente al Estudio Técnico PER en 2011 (IDAE, 2011), es sin duda la más completa y precisa, aunque al ser a nivel nacional la escala del estudio impide obtener resultados detallados y precisos, si bien no se observa una gran desviación respecto a datos publicados más recientemente. Esta proporciona para la mayor parte del territorio un potencial de $60-80 \mathrm{~W} / \mathrm{m}$, siendo de 35-60 W/m en algunas zonas dispersas por todo el territorio gallego y $>80 \mathrm{~W} / \mathrm{m}$ para algunas zonas del centro sur de la provincia de Ourense y Centro-norte de la de Lugo. Hay que mencionar que para esta estimación se toman los valores de capacidad de extracción de calor de las rocas de la norma VDI4640, de modo que se establece para el granito una capacidad de 68-85 $\mathrm{W} / \mathrm{m}$, para rocas básicas $40-65 \mathrm{~W} / \mathrm{m}$ y para gneis $70-85 \mathrm{~W} / \mathrm{m}$. El estudio considera también las aguas subterráneas y establece que en Galicia existen zonas aisladas con potencial recurso geotérmico de baja temperatura y zonas con posible aprovechamiento por existencia de potenciales consumidores, con temperaturas de $50-90^{\circ} \mathrm{C}$ a profundidades de 500-2000 m. También establece en otras zonas recursos geotérmicos de media temperatura reconocidos o estimados, en las cuatro provincias de Galicia, con temperaturas de $110-130^{\circ} \mathrm{C}$ a profundidades de $1500-2000 \mathrm{~m}$. Además, establece algunos recursos de alta temperatura en algunos puntos de la provincia de Ourense, y Pontevedra, la ciudad de Lugo y Arteixo en A Coruña. El estudio establece también cálculos para la evaluación de recursos geotérmicos ligados a posibles Sistemas Geotérmicos Estimulados (EGS) en algunas zonas.

\subsection{Nuevos datos para una reevaluación del potencial geotérmico}

A pesar de que no hay estudios posteriores a 2011, existen datos que han sido publicados posterior y anteriormente que no han sido considerados en la estimación de 2011 y que permiten, al menos, reevaluar el potencial geotérmico o comprobar hasta cierto punto si la estimación es exacta en algunos aspectos. Por una parte, en 2001 fueron publicados los resultados del Proyecto MARNA (2001). Este proyecto consistió en evaluar la radiación gamma generada por el subsuelo en toda España y resultó además en la publicación del Mapa de Radiación 
Gamma de Galicia. Dado que la principal fuente de calor en rocas de la corteza son los radioisótopos de $\mathrm{U}$, Th y $\mathrm{K}$, y que estos son los principales responsables de la emisión de radiación gamma del subsuelo, existe la posibilidad de evaluar la generación de calor a partir de la tasa de radiación medida (Birch, 1954; Rybach, 1976, 1986; Bücker y Rybach, 1996). Por tanto, los datos del proyecto permiten al menos contrastar las evaluaciones realizadas del potencial a partir de datos petrológicos e hidrológicos de tipo genérico y no específicos de la zona de estudio. Adicionalmente, se ha publicado el mapa nacional de Potencial de Radón de España (CSN, 2017). En este mapa se estima una elevada liberación potencial de $\mathrm{Rn}$ en la mayor parte del Territorio de Galicia. El Rn es un gas noble radiactivo que se produce a partir de ${ }^{238} \mathrm{U}$ principalmente $\left({ }^{222} \mathrm{Rn}\right)$ y de ${ }^{232} \mathrm{Th}$ $\left({ }^{220} \mathrm{Rn}\right)$. Estos radioisótopos primordiales, y especialmente el ${ }^{238} \mathrm{U}$, son los principales generadores de calor en rocas. Esto implica una elevada concentración de U en las rocas en donde el potencial de $\mathrm{Rn}$ es elevado.

Por último, en 2013 se ha publicado el Atlas Geoquímico de España (IGME, 2012). En este Atlas se han elaborado mapas de España para la distribución y concentración de la mayor parte de elementos de la tabla periódica. La metodología empleada en este atlas ha consistido en la toma de un gran número de muestras (número total) repartidas por todo el territorio nacional, tanto de sedimento como de suelos (en horizontes superiores e inferiores) que una vez seleccionadas y tratadas han sido analizadas, dependiendo del elemento de interés, por varios métodos: Espectrometría de Masas con Plasma Acoplado Inductivamente(ICPMS), espectroscopia de emisión atómica de plasma acoplado por inducción (ICP-AES), análisis instrumental por activación de neutrones (INNA). Entre los elementos analizados están el K, U y Th. Si bien los elementos analizados corresponden a la suma de los isótopos presentes en las muestras, es posible estimar, a partir de estos datos las proporciones correspondientes de cada uno de los isótopos padres a partir de esto, dado que sus proporciones son bastante constantes en la naturaleza. En la naturaleza los isótopos del $\mathrm{K}$ que constituyen casi el 100\% del $\mathrm{K}$ total son el ${ }^{39} \mathrm{~K}(93,26 \%)$, el ${ }^{40} \mathrm{~K}$ y el ${ }^{41} \mathrm{~K}(6,73 \%)$. De éstos solo el ${ }^{40} \mathrm{~K}$ es inestable y supone un $0,012 \%$ del $\mathrm{K}$, lo que permite calcular su concentración a partir del $\mathrm{K}$ total. En el caso del uranio, 3 isótopos inestables constituyen el $100 \%$ de este elemento, el ${ }^{234} \mathrm{U},{ }^{235} \mathrm{U}$ y ${ }^{238} \mathrm{U}$, siendo la abundancia del ${ }^{238} \mathrm{U}$ del $99,27 \%$ y la del ${ }^{235} \mathrm{U}$ del $0,72 \%$, lo que permite estimar sus concentraciones a partir de la concentración total de U. El torio tiene hasta 5 isótopos, pero el ${ }^{232} \mathrm{Th}$ supone casi el $100 \%$ de total. Por tanto, los datos del Atlas permiten estimar la concentración de radioisótopos primordiales en las muestras analizadas, sin considerar cuestiones relativas a las desviaciones posibles debidas al método de análisis. En el caso de la ICPAES e ICP-MS los procedimientos químicos de preparación de muestra pueden producir esas desviaciones dependiendo de la composición mineral de las muestras tratadas. Independientemente de este factor, los datos del atlas no son directamente atribuibles a las rocas del sustrato de las zonas muestreadas. La composición química de los sedimentos y suelos analizados no es extrapolable a la roca subyacente aunque en algunos casos puede proporcionar una idea de su composición, y también de su contenido en los radioisótopos citados. Por una parte, la formación de un suelo en una superficie de 
roca implica procesos de meteorización física, química y biológica. Estos procesos pueden producir concentraciones o pérdidas de algunos de estos elementos, y sobre todo del $\mathrm{K}$ y del U debido a que ambos son móviles en condiciones oxidantes y en un rango amplio de pH. Además, si los suelos se desarrollan sobre sedimentos, los datos geoquímicos obtenidos no serán necesariamente representativos de la roca, siempre y cuando el sedimento tenga un origen alóctono.

Por otra parte, los procesos de meteorización química y formación de elementos suelen implicar la pérdida y concentración de otros elementos que tienen relación con el U y Th. El ${ }^{238} \mathrm{U},{ }^{235} \mathrm{U}$ y ${ }^{232} \mathrm{Th}$ se desintegran dando lugar a cadenas de desintegración en las que se generan radioisótopos que a su vez se desintegran generando otros dando lugar a una cascada de radioisótopos con diferentes períodos y tipos de desintegración (alfa o beta), y propiedades químicas. Esto hace que algunos puedan ser fácilmente movilizados (U, Ra), liberados en forma de gas $(\mathrm{Rn})$ o permanezcan inmóviles (Th) en las cadenas. Si esto no se produce, se dice que las cadenas están en equilibrio secular, pero si se produce estarán en desequilibrio. Esto hace que no se pueda estimar la liberación de calor generada por esas cadenas a partir simplemente del dato de U o Th de padres. Esto será una causa de error no aleatorio en las estimaciones que solo es corregible si se mide la concentración de $\mathrm{U}$ y $\mathrm{Th}$ en la roca basal, dado que este desequilibrio no existe en las rocas a menos que algún proceso de meteorización lo haya producido recientemente (en los últimos miles de años).

En cualquier caso, es posible utilizar los datos obtenidos en el Atlas con el fin de obtener una estimación, aunque imprecisa y relativamente inexacta de la concentración de radioisótopos en las rocas del sustrato de Galicia.

\section{DESCRIPCIÓN GEOLÓGICA DEL ÁREA DE ESTUDIO}

El marco geológico en el que se encuentra la Comunidad Autónoma de Galicia se compone fundamentalmente de los materiales precámbricos y paleozoicos del Macizo Ibérico con alguna cuenca sedimentaria cenozoica. En esta región podemos encontrar las rocas más antiguas de la Península Ibérica. El Macizo Ibérico o Hespérico se forma durante la Orogenia Varisca, conformando el relieve emergido más antiguo de la Península Ibérica y el afloramiento más occidental del Orógeno Varisco Europeo. (Vera, 2004). El Macizo Ibérico presenta rasgos propios de un orógeno de colisión, con mayor deformación, presencia de metamorfismo y magmatismo en la zona central, y unas zonas externas en las que la deformación es menor y en la que los materiales metamórficos e ígneos tienen menor presencia o incluso no aparecen. Dentro de este macizo se han establecido zonas con características estratigráficas, estructurales, de metamorfismo y de magmatismo diferentes (Lozte, 1945; Julivert et al., 1974; Farias et al., 1987).

En relación a las zonas en las que se divide este Macizo Ibérico, en Galicia se pueden encontrar rocas pertenecientes a tres de ellas. En la parte oriental, encontramos la Zona Asturoccidental-Leonesa (ZAOL), compuesta por materiales paleozoicos de origen sedimentario. En la parte central y occidental, encontramos la Zona Centroibérica (ZCI) en la que predominan materiales paleozoicos y precámbricos con intrusiones anteriores al Pérmico de granitoides de distintas épocas, tanto pre-, 
como sin- y postcinemáticos respecto a la Orogenia Hercínica (Martínez-Catalán et al., 2004). Al Oeste se encuentra la Zona de Galicia Tras os Montes (Farias, 1987, Arenas et al., 2004) compuesto por diversos complejos polimetamórficos con materiales ígneos básicos y ultrabásicos con distintos grados de deformación y metamorfismo. Constituye una lámina alóctona emplazada sobre rocas del Precámbrico al Paleozoico inferior de la ZCI. Por ello, en Galicia se encuentran numerosos afloramientos de rocas graníticas con diversas tipologías y edades de emplazamientos, siendo más importantes los afloramientos dentro del Dominio de Galicia Tras os Montes. Cabe destacar la presencia de magmatismo granítico prevarisco y varisco en la ZAOL, aunque en Galicia estos granitos son sin o postectónicos. Dentro de la Zona Galicia Tras Os Montes, en la parte occidental de Galicia existe una gran profusión de magmatismo granítico (Cuesta, Gallastegui, 2004), pudiendo establecerse 3 tipologías que se agrupan de modo secuencial: granitos calcoalcalinos sincinemáticos, granitos peralumínicos sin a postcinemáticos y granitos calcoalcalinos postcinemáticos. Estos serían, en principio, los más interesantes en el estudio del potencial geotérmico, sin descartar algunos materiales paleozoicos de origen sedimentario en la zona oriental.

Una breve descripción de estos tres tipos de granito muestra que todas presentan variaciones en las facies graníticas presentes. En los granitos calcoalcalinos sincinemáticos predominan granodioritas y monzogranitos y se pueden hallar rocas intermedias e incluso básicas relacionados con su intrusión y con valores altos de $\mathrm{K}$ (Castro et al., 2002). Para los granitos peralumínicos, la variedad litológica y textural es mayor.
También se observan otras rocas asociadas como migmatitas, ortogneises y metasedimentos ligados a los procesos anatécticos corticales variscos (Bellido et al., 1987). En los granitos postcinemáticos predominan monzogranitos de diversos tipos y se hallan elementos propios de protolito cortical en los que el proceso de evolución magmática principal ha sido la cristalización fraccionada (Cuesta, 1991).

\section{DATOS Y MÉTODOS DE ESTI- MACIÓN DEL POTENCIAL GEOTÉR- MICO}

Si consideramos que la capacidad de generación de calor geotérmico en rocas depende principalmente de su contenido en los radioisótopos antes citados $\left({ }^{238} \mathrm{U}\right.$ y ${ }^{232} \mathrm{Th}$ y sus respectivas progenies y ${ }^{40} \mathrm{~K}$ ) se puede estimar en parte su potencial geotérmico a partir de este. Por supuesto existen otros factores que influyen de forma determinante en el potencial geotérmico como la densidad de la roca, o su conductividad térmica entre otros (permeabilidad y fracturación, etc.), pero en los casos en los que no existen anomalías térmicas por factores geológicos diferentes a los geoquímicos y petrológicos (como por ejemplo la existencia de fallas activas, anomalías térmicas, etc.), los radioisótopos primordiales de la roca son el principal generador de calor. De esta manera, rocas con elevadas concentraciones de estos radioisótopos tendrán un mayor potencial geotérmico que las que contienen una menor concentración.

Las concentraciones de estos radioisótopos dependen de una serie de factores que hacen que su distribución se pueda relacionar con cada tipo de roca, aunque existen factores que hacen que su distribución sea 
relativamente heterogénea, por lo que es necesario estudiar la roca in situ para obtener una buena estimación. Durante la segunda mitad del siglo XX, el uso de la energía nuclear tanto con el propósito de obtener energía como de su uso armamentístico llevó a una extensa investigación a nivel mundial acerca de la geoquímica de algunos de estos elementos, principalmente del $\mathrm{U} y$ del Th. Gracias a este trabajo se conocen los rangos habituales de concentración de estos radioisótopos en diferentes tipos de roca y las principales causas de su variación (Boyle, 1982, Gascoyne, 1992). Estos rangos han sido utilizados para estimar el potencial geotérmico de las rocas a escala regional o nacional, en base a los rangos más frecuentes de cada tipo de roca, sin la necesidad de realizar análisis de cada roca estudiada. Sin embargo, esos rangos son muy variables para algunos tipos de roca y en particular para algunas rocas magmáticas como los granitos. Dada la abundancia de esta roca en Galicia, y la variedad de granitoides, debe valorarse la posibilidad de que el uso de estos valores tabulados para obtener este potencial conduzca a obtener resultados inexactos. Como medida de contraste de esas estimaciones pueden utilizarse todos los datos existentes en la actualidad que se resumen en: datos relativos a la radiactividad de rocas, y datos relativos a la geoquímica de rocas. Este trabajo, se centra en los primeros.

En el caso de los primeros, el Mapa de Radiación Gamma de Galicia (MARNA, 2001) constituye una buena herramienta para obtener información de la concentración de radioisótopos en rocas, dado que la tasa de radiación gamma de las rocas del subsuelo depende fundamentalmente de la presencia de U, Th y K en ellas. De hecho, la tasa de radiación gamma es una de las herramientas que se utilizan en la exploración geotérmica (Birch, 1954; Rybach, 1976). Bien es cierto, que los tres radioisótopos en cuestión no tienen el mismo efecto en la generación de ese potencial. De los tres, el U es el de mayor interés, y su concentración es la que más directamente está relacionada con el potencial geotérmico de una roca. Existen también un mapa de potencial de $\mathrm{Rn}$ en España y en Galicia (CSN, 2017). Estos mapas asignan valores muy elevados de generación y liberación de este gas en la mayor parte del territorio gallego, y en especial en las zonas de litología granítica. El Rn está principalmente compuesto por el ${ }^{222} \mathrm{Rn}$ que es un isótopo formado por desintegración del ${ }^{222} \mathrm{Ra}$, componente de la serie de desintegración del ${ }^{238}$ U. Por lo tanto, su potencial liberación, aunque depende de otros factores también, está directamente relacionada con la concentración de $\mathrm{U}$, es decir, allí donde existe liberación de radón existe un cierto potencial geotérmico. En el trabajo que se presenta aquí, aunque ambos datos pueden considerarse, se utiliza principalmente la tasa de radiación gamma para revisar la estimación el potencial geotérmico de Galicia publicada en IDAE (2011).

Por otra parte, la publicación del Atlas Geoquímico de España (IGME, 2012) proporciona estimaciones del U, Th y K presente en el suelo. Dada la ausencia de grandes depósitos sedimentarios en la mayor parte del territorio gallego, la presencia de estos radioisótopos en el suelo, puede relacionarse con la roca subyacente, y por tanto proporcionan una estimación, aunque sesgada por diferentes procesos de meteorización, de éstos en la roca. Estos datos permiten estimar, a priori, si la concentración de estos radiotisótopos en la roca corresponde a los valores 
típicos esperados en rocas o no. En el caso de rocas graníticas, esto es de especial interés, dado que los rangos son muy amplios y para $\mathrm{U}$ y $\mathrm{Th}$ oscilan entre concentraciones de pocos ppm hasta unos 100 ppm (Boyle, 1982). Esto supone una gran variabilidad en el potencial geotérmico que depende de las características petrológicas y geoquímica de cada tipo de granito.
Para aplicar ambos tipos de datos se han utilizado dos aproximaciones. Por una parte, a partir de los datos del Atlas Geoquímico de España (IGME, 2012) se ha estimado la producción de calor geotérmico (Radiogenic Heat Production o $H P$ ) propuesto por Birch (1954) y modificado posteriormente por Rybach (1976) y Lee et al. (1984). Esta estimación sigue la siguiente ecuación:

$$
H P\left(\mu \mathrm{Wm}^{-3}\right)=\rho\left(0.035 C_{\mathrm{K}}+0.097 C_{\mathrm{U}}+0.026 C_{\mathrm{Th}}\right)
$$

donde $\rho$ corresponde a la densidad de la roca estudiada (en $\mathrm{kg} \mathrm{m}^{-3}$ ) y $C_{\mathrm{K}}, C_{\mathrm{U}}$ y $C_{\mathrm{Th}}$ corresponden a las concentraciones de $\mathrm{K}$ (\%), U (ppm) y Th (ppm), respectivamente. Para este índice de estimación, se considera que valores $4 \mu \mathrm{W} / \mathrm{m}^{3}$ indican una elevada producción de calor, por lo que la roca estudiada se podría considerar un recurso con potencial económico para el uso de energía geotérmica (McCay et al., 2014).

$$
A\left[\mathrm{pW} / \mathrm{m}^{\prime}\right]=0,0158(G R[\mathrm{API}]-0.8)
$$

siendo $G R$ el registro de radiación gamma natural (o natural gamma log), y (API) el resultado de medir la radiación gamma en unidades API (American Petroleum Institute). Las unidades API se definen como el número total de cuentas registrados de la actividad gamma de una pizarra media considerando que contiene 2,66 ppm de U, 12,3 ppm de Th y un 3,2\% de $\mathrm{K}$, de modo que esta concentración correspondería a 121,7 API (Ferti et al., 1987). Teniendo en cuenta esta equivalencia, y utilizando esas concentraciones de elementos se obtiene una tasa de dosis de 79,1 nGy/h, lo que equivale $7,9 \mathrm{mR} / \mathrm{h}$, que es la unidad utilizada por el mapa de radiación gamma de Galicia. Dado que la ecuación (2) permite estimar la pro-
Por otra parte, el uso de los datos de radiación gamma ambiental obtenidos para la superficie de Galicia permite realizar una estimación menos precisa pero que permite contrastar la anterior en base a la propuesta de Rybach (1976). Esta propuesta es especialmente adecuada para rocas graníticas Bücker y Rybach (1996). Según esta propuesta se puede estimar la producción de calor geotérmico $(A)$ a partir de la expresión:

ducción de calor geotérmico $(A)$ a partir de los API obtenidos, se puede estimar así la producción de calor geotérmico a partir de la tasa de radiación gamma medida en $\mathrm{mR} / \mathrm{h}$ en el mapa de radiación gamma de Galicia (CSN, 2001), si bien esta estimación es poco precisa y exacta considerando el método utilizado para medir esa radiactividad y los factores que afectan a este tipo de mediciones. En particular, las medidas de radiación gamma realizadas en superficie, a un metro del suelo, se ven influenciadas por la humedad del aire y del suelo, la vegetación, la presencia de materia orgánica en el suelo, la topografía y otros factores. Sin embargo, los datos sirven para obtener una aproximación rápida al potencial en Galicia. 


\section{RESULTADOS Y DISCUSIÓN}

Considerando los rangos de concentraciones de radioisótopos primordiales $\left({ }^{40} \mathrm{~K}\right.$, ${ }^{232} \mathrm{Th}$ y ${ }^{238} \mathrm{U}$ ) en cada tipo de roca, referidas en la bibliografía (Boyle, 1982; Gascoyne, 1992; Sanjurjo-Sánchez y Alves, 2017), y teniendo en cuenta los tipos de roca mayoritariamente existentes en el territorio gallego, se puede hacer una estimación del rango de producción de calor geotérmico (HP) esperable en Galicia en base a dichos datos. Estos datos pueden ser, a su vez, comparados con los datos obtenidos a través del Atlas Geoquímico de España. De esta forma, los rangos esperados para algunos de los tipos de roca más habituales en Galicia se exponen en la tabla 1.
A partir de estos de concentración en \% para el K y ppm para Th y U es posible estimar la actividad de los radioisótopos mayoritarios $\left({ }^{40} \mathrm{~K},{ }^{232} \mathrm{Th} \mathrm{y}{ }^{238} \mathrm{U}\right)$, cuyos valores esperados y calculados, considerando equilibrio secular en las series de ${ }^{232} \mathrm{Th}$ y ${ }^{238} \mathrm{U}$, dado que es lo más habitual en rocas, se pueden observar en la misma tabla 1. Igualmente, es posible estimar el rango de producción de calor geotérmico o HP para cada uno de los tipos de roca considerados (tabla 1) a partir de la ecuación (1). Estos datos, indican qué si bien el potencial geotérmico es bajo en una gran parte de rocas, como gneis, esquistos o rocas básicas o ultrabásicas, en algunas pizarras y sobre todo en granitoides es potencialmente alto.

Tabla 1. Rangos de concentración de radioisótopos primordiales y producción de calor geotérmico esperada en los tipos de roca más habituales en Galicia.

\begin{tabular}{|l|c|c|c|c|}
\hline Roca & $\mathrm{Th}(\mathrm{ppm})$ & $\mathrm{U}(\mathrm{ppm})$ & $\mathrm{K}(\mathrm{ppm})$ & $H P\left(\mu \mathrm{Wm}^{-3}\right)$ \\
\hline Granitos & $4,2-100$ & $01,5-100$ & $2,3-9$ & $0.9-33.4$ \\
\hline Pizarras & $9-20$ & $2-7$ & $2-7$ & $1,3-3,8$ \\
\hline Gneiss & $7-15$ & $3-6$ & $2-7$ & $1,4-3,2$ \\
\hline Esquistos & $5-12$ & $1-3$ & $2-6$ & $0,8-2,2$ \\
\hline Diorita, gabro, anfibol & $0,04-4$ & $0,001-1$ & $0,03-2$ & $0,0-0,7$ \\
\hline
\end{tabular}

En el caso de Galicia, es necesario resaltar la elevada proporción de territorio en la que se encuentran rocas magmáticas, y particularmente graníticas, donde la variabilidad en la concentración de estos radioisótopos es grande. Esta variabilidad se considera según el carácter félsico o máfico de estas rocas en la tabla 2, en la que se exponen datos calculados para algunas de las principales rocas existentes en Galicia a partir de los datos de las referencias recopiladas por SanjurjoSánchez y Alves (2017). En esta tabla, y a partir de los rangos expuestos se estima también la producción de calor geotérmico para cada tipo de roca magmática. Se puede observar como el HP es sensiblemente superior para rocas con una composición félsica o incluso intermedia.

Por supuesto, los rangos obtenidos son genéricos, pero indican que el HP es tremendamente variable, lo que desvela la necesidad de estudiar la composición de cada roca y estimar el HP de cada una para obtener una buena estimación. Este tipo de datos no existen en la actualidad para todos los tipos de roca de Galicia, o no están disponibles, si bien, algunos estudios parciales permiten obtenerlos para algunas zonas y tipos de roca. 
Tabla 2. Rangos de concentración de radioisótopos primordiales y producción de calor geotérmico esperada en rocas magmáticas presentes en Galicia.

\begin{tabular}{|l|c|c|c|c|}
\hline & $\mathrm{Th}(\mathrm{ppm})$ & $\mathrm{U}(\mathrm{ppm})$ & $\mathrm{K}(\mathrm{ppm})$ & $H^{\prime}\left(\mu \mathrm{Wm}^{-3}\right.$ \\
\hline Granito, sienita & $4,2-23$ & $1,5-13$ & $2,3-4,5$ & $0,9-5,3$ \\
\hline Intermedio & $1,0-7,0$ & $4,0-19$ & $1,7-3,7$ & $1,3-5,7$ \\
\hline Mafico (gabro) & $1,0-7$ & $0,3-1,6$ & $0,5-1,8$ & $0,2-1,1$ \\
\hline Ultramafico (peridotita) & $0,004-0,1$ & $0,001-0,03$ & $0,03-0,5$ & $0,0-0,1$ \\
\hline
\end{tabular}

Una alternativa que permite hacer una estimación imprecisa de todo el territorio es usar los datos existentes en el Atlas Geoquímico de España, y en concreto aquellos derivados de los horizontes inferiores del suelo, para hacer una estimación del contenido de los mismos radioisótopos en las rocas. En el Atlas, estos datos se obtienen del horizonte $\mathrm{C}$, que corresponde al regolito formado por alteración de la roca. Esto implica que las concentraciones no corresponden exactamente a la de la roca, dado que algunos procesos de meteorización química afectan a las concentraciones de K y U. Sin embargo, pueden considerarse indicativos $\mathrm{O}$ aproximativos de la concentración de estos radioisótopos en las rocas subyacentes de los citados regolitos. A partir de estos datos, y con los rangos estipulados en el Atlas se pueden obtener rangos que se detallan en la tabla 3. A partir de estos rangos de concentraciones se pueden obtener los HP mínimos y máximos esperables que se han agrupado por provincias y se detallan en la misma tabla. Una vez más puede observarse, por una parte, la enorme variabilidad del $H P$ en cada provincia y los elevados valores máximos que se pueden obtener, que implicarían un elevado potencial geotérmico, si bien también es razonable remarcar que la gran variabilidad del potencial requiere un estudio de detalle de cada tipo de roca. Una observación rápida de estos rangos indica un mayor $H P$ en las provincias con mayor cantidad de granito, del tipo y composición que sea, frente a un menor $H P$ en las provincias con mayor cantidad de rocas básicas, ultrabásicas o metasedimentarias.

Tabla 3. Rangos de concentración de radioisótopos primordiales y producción de calor geotérmico en rocas de las provincias de Galicia.

\begin{tabular}{|l|c|c|c|c|}
\hline Provincia & $\mathrm{K}(\%)$ & $\mathrm{Th}(\mathrm{ppm})$ & $\mathrm{U}(\mathrm{ppm})$ & $H P\left(\mu \mathrm{Wm}^{-3}\right)$ \\
\hline A Coruña & $0,78-3,2$ & $2,7-40,4$ & $7,6-148$ & $2,2-41,3$ \\
\hline Lugo & $0,78-3,2$ & $2,1-5-5$ & $5,2-36$ & $1,6-12,3$ \\
\hline Pontevedra & $2,1-5-5$ & $5,4-40,4$ & $17,3-148$ & $4,7-38,9$ \\
\hline Ourense & $2,1-5,5$ & $5,4-40,4$ & $13,4-148$ & $4,0-41,5$ \\
\hline
\end{tabular}

La última alternativa para evaluar los $H P$ obtenidos por los dos modos anteriores es considerar los datos del mapa de Radiación Gamma de Galicia (MARNA, 2001). Esta 
radiación gamma de fondo se debe principalmente a la presencia de ${ }^{238} \mathrm{U},{ }^{232} \mathrm{Th}$ y ${ }^{40} \mathrm{~K}$ en las rocas del substrato y del suelo, de modo que pueden ser considerados para evaluar el $H P$ de las rocas de cada zona considerando la ecuación (2). Teniendo en cuenta estos datos y la variabilidad en la tasa de dosis gamma en cada provincia se obtienen los datos especificados en la tabla 4. En esta misma tabla se especifican los HP obtenidos a partir de estos datos. Nuevamente, se observa una gran variabilidad en cada provincia y un probable elevado $H P$, de modo que sería recomendable, como ya se ha mencionado antes, realizar estudios en detalle, y en concreto medidas de la radiación gamma en el sustrato para confirmar estas estimaciones.

Tabla 4. Tasas de dosis de radiación gamma (DR - Dose rate) en cada provincia de Galicia y rangos de HP estimados en función de ésta.

\begin{tabular}{|l|c|c|c|}
\hline Provincia & $D R \min (\mu \mathrm{R} / \mathrm{h})$ & $D R \max (\mu \mathrm{R} / \mathrm{h})$ & $H P\left(\mu \mathrm{Wm}^{-3}\right)$ \\
\hline A Coruña & 6 & 30 & $1,3-6,6$ \\
\hline Lugo & 8,0 & 35,0 & $1,7-7,7$ \\
\hline Pontevedra & 10,0 & 35,0 & $2,2-7,7$ \\
\hline Ourense & 10,0 & 35,0 & $2,2-7,7$ \\
\hline
\end{tabular}

Por supuesto, la evaluación realizada aquí ha consistido en valorar únicamente la concentración de los principales radionúclidos primordiales en la roca, dado que son los principales generadores de calor en ellas. Sin embargo, es todavía necesario valorar otras cuestiones como la conductividad y difusividad térmica. En granitos, es bien conocido que la conductividad térmica es elevada, lo que convierte a estas rocas en especialmente interesantes para su uso en Geotermia de Baja entalpía.

\section{CONCLUSIONES}

A pesar de que desde los años 70 y 80 se han realizado y publicado varios estudios que permiten estimar el potencial geotérmico en la comunidad autónoma de Galicia, estos han sido generales, dado que se han utilizado datos geológicos pero no datos geoquímicos específicos de cada zona de estudio. En este trabajo se ha realizado una estimación del potencial geotérmico de algunas rocas de Galicia en base a datos conocidos de radiación gamma y de la composición química de rocas, publicados en forma de cartografía en las últimas dos décadas. A partir de estos datos, se puede observar que las estimaciones realizadas han sido muy conservadoras, tendiendo a infravalorar este recurso. En este trabajo, se ha mostrado que esta estimación del potencial a partir de dos tipos de datos son coincidentes, observándose una gran variabilidad entre rocas y zonas, por lo que se considera necesario llevar a cabo un estudio pormenorizado por zonas del potencial geotérmico para realizar una correcta evaluación de éste. Solamente de esta manera será posible un adecuado desarrollo de este aprovechamiento energético en la comunidad, dado que una información detallada de este tipo permitiría realizar estudios de 
aprovechamiento para cada zona, planificando el uso de esta energía incluso en planes urbanísticos.

\section{REFERENCIAS}

ACLUXEGA (2012) Uso de la geotermia para la climatización de edificio. Jornadas de Bioconstucción Porto do Molle (Nigrán).

Arenas, R.; Martínez-Catalán, J. R. y Díaz García, F. (coords.) (2004). «Zona de Galicia-Trás-os-Montes». En Vera Torres, J. A. Geología de España. Sociedad Geológica de España e Instituto Geológico y Minero de España. pp. 133165. ISBN 84-7840-546-1.

Bellido Mulas, F.; González Lodeiro, F.; Klein, E.; Martínez Catalán, J.R.; Pablo Macía, J. G. (1987) Las rocas graníticas hercínicas del norte de Galicia y occidente de Asturias. Memorias Instituto Geológico y Minero de España, t. 101, Madrid, $157 \mathrm{p}$.

Birch, F. Heat from radioactivity. In Nuclear Geology; Wiley: New York, NY, USA, 1954; pp. 148-174.

Boyle, R.W. (1982) Geochemical propecting for thorium and uranium deposits. Elsevier, New York, 489pp.

Bücker, C., Rybach, L. (1996) A simple method to determine heat production from gamma-ray logs. Marine and Petroleum Geology, 13 (4), 373-375. https:// doi.org/10.1016/0264-8172(95)00089-5

Castro, A.; Corretgé, L. G.; De la Rosa, J. D.; Enrique, P.; Martínez, F. J.; Pascual, E.; Lago, M.; Arranz, E.; Galé, C.; Fernández, C.; Donaire, T y López, S. (2002): Paleozoic magmatism. En The Geology of Spain, W. Gibbons y T. Moreno. Eds.) Geol. Soc. (London). 117-153
CSN (2001) Proyecto Marna. Mapa de radiación gamma natural. Informes Técnicos. Consejo de Seguridad Nuclear.

CSN (2017) Mapa del potencial de Radón de España. Actualización de enero de 2017. Consejo de Seguridad Nuclear.

Cuesta, A: (1991) Petrología granítica del plutón de Caldas de Reyes (Pontevedra, España) Estructura, mineralogía, química y petrogénesis. Nova Terra, O Castro, 5. $363 \mathrm{p}$.

Cuesta, A., Gallastegui, G. (coords.) (2004) Galicia Occidental En Vera Torres, J. A. Geología de España. Sociedad Geológica de España e Instituto Geológico y Minero de España. pp. 133-165. ISBN 847840-546-1.

Farias, P.; Gallastegui, G.; González Lodeiro, L.; Marquínez, J.; Martín Parra, L. M.; Martínez Catalán, J. R.; Pablo Maciá, J. G. \& Rodríguez Fernández, L. R. (1987) Aportaciones al conocimiento de la litoestratigrafia y estructura de Galicia Central. Mem. Mus. Lab. miner. geol. Fac. Ciênc. Univ. Porto, 1: 411431.

Ferti, W. H. (1987) Log-Derived Evaluation of Shaly Clastic Reservoirs; Journal of

175-194.

Gascoyne, M. (1992) Geochemistry of the actinides and their daughters. In: Ivanovich, M., Harmon, R.S. (eds.) Uranium-series disequilibrium: applications to Earth, marine, and environmental sciences. Clarendon Press, Oxford, pp. 34-61.

GEOPLAT (2015) Análisis del sector de la energía geotérmica en España. GEOPLAT, Plataforma Tecnológica Española de Geotérmia.

IDAE (2011) Evaluación del potencial de energía geotérmica. Estudio Técnico 
PER 2011-2020. Instituto para la Diversificación y Ahorro de Energía.

IGME (1975) Inventario General de Manifestaciones Geotérmicas en el territorio nacional. Plan Nacional de Mineria. Ministerio de Industria.

IGME (1980) Estudio de las manifestaciones termales de Galicia, orientadas a su posible explotación como recursos geotérmicos. Ministerio de Industria y Energía.

IGME (2012) Atlas Geoquímico de España. Ministerio de Economía y Competitividad.

Julivert , M; Fontbote, J.M.; Ribeiro, A. Conde, I: (1974). Mapa Tectónico de la Península Ibérica y Baleares. E: 1/1.000.000. IGME, 113P.

Lee, M.; Wheildon, J.; Webb, P.; Brown, G.; Rollin, K.; Crook, C.; Smith, I.; King, G.; Thomas-Betts, A. Hot dry rocks prospects in caledonian granites: Evaluation of results from the bgs-ic-ou research programme (1981-1984). En Investigation of the Goethermal Potential of the UK; British Geological Survey: Keyworth, UK, 1984.

Lotze, F. (1945). Zur gliederung der Variszichen der lberischen Meseta. Geotektonísche Forschungen, 6, 78-92. Traduc. Ríos, J. M.: Observaciones respecto a la división de las variscides de la Meseta Ibérica. Publ. Extr. Geol. Esp., 5, 149166, 1950.

MARNA, (2001). Proyecto MARNA. Mapa de radiación gamma natural. Consejo de Seguridad Nuclear.

Martínez-Catalán, J. R.; Martínez Poyatos, D. y Bea, F. (coords.) (2004). «Zona Centroibérica». En Vera Torres, J. A. Ge- ología de España. Sociedad Geológica de España e Instituto Geológico y Minero de España. pp. 68-133. ISBN 84-7840546-1.

McCay, A.T., Harley, T.L., Younger, P.L., Sanderson, D.C.W., Cresswell, A.J. (2014) Gamma-ray spectrometry in geothermal exploration: state of the art techniques. Energies, 7(8), 4757-4780. https://doi.org/10.3390/en7084757

Orche García, E. (2011) Energía geotérmica. ETSI Minas - Universidad Politécnica de Madrid. Madrid.

Pous, J., Jutglar, L. (2004) Energía geotérmica. Ed. CEAC. Barcelona.

Pollack, H.N., Hurter, S.J., Johnson, J.R. (1993) Heat flow from the Earth's interior: analysis of the global data set. Reviews of Geophysics, 31(3), 267-280. https://doi.org/10.1029/93RG01249

Rybach, L. (1976) Radioactive heat production: A physical property determined by the chemistry of rocks. In The Physics and Chemistry of Minerals and Rocks; Stems, R.G.J., Ed.; Wiley-Interscience: New York, USA, 1976; pp. 309-318.

Rybach, L. (1986) Amount and significance of radioactive heatsources in sediments. In: ThermalModelling in SedimentaryBasins, Collections Colloques et Seminaires (Ed J. Burrus) 44,Editions Technip, Paris

Sanjurjo-Sánchez, J., Alves, C. (2017) Geologic materials and gamma radiation in the built environment. Environmental Chemistry Letters, 15 (4), 561-589. https://doi.org/10.1007/s10311-017-0643-1

Vera, J.A, editor (2004): Geología de España. SGE-IGME, Madrid, ISBN 847840-546-1. $890 \mathrm{p}$. 